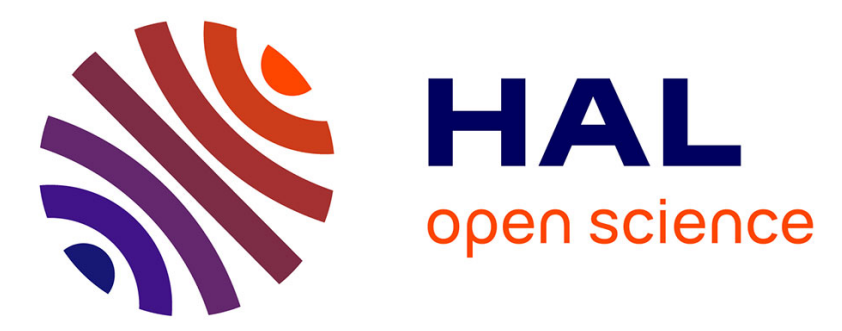

\title{
Du nomadisme idéologique à l'allégeance partisane : les mondes franco-vénézuéliens de la réélection de Hugo Chávez (2012)
}

Fabrice Andréani

\section{- To cite this version:}

Fabrice Andréani. Du nomadisme idéologique à l'allégeance partisane : les mondes franco-vénézuéliens de la réélection de Hugo Chávez (2012). Critique Internationale, 2013, 59 (2), pp.119-132. 10.3917/crii.059.0119 . halshs-02022947

\section{HAL Id: halshs-02022947 https://shs.hal.science/halshs-02022947}

Submitted on 18 Feb 2019

HAL is a multi-disciplinary open access archive for the deposit and dissemination of scientific research documents, whether they are published or not. The documents may come from teaching and research institutions in France or abroad, or from public or private research centers.
L'archive ouverte pluridisciplinaire HAL, est destinée au dépôt et à la diffusion de documents scientifiques de niveau recherche, publiés ou non, émanant des établissements d'enseignement et de recherche français ou étrangers, des laboratoires publics ou privés. 


\title{
DU NOMADISME IDÉOLOGIQUE À L'ALLÉGEANCE PARTISANE : LES MONDES FRANCO-VÉNÉZUÉLIENS DE LA RÉÉLECTION DE HUGO CHÁVEZ (2012)
}

\author{
Fabrice Andréani \\ Presses de Sciences Po | Critique internationale
}

\author{
2013/2 - N०59 \\ pages 119 à 132
}

ISSN 1290-7839

Article disponible en ligne à l'adresse:

http://www.cairn.info/revue-critique-internationale-2013-2-page-119.htm

Pour citer cet article :

Andréani Fabrice, « Du nomadisme idéologique à l'allégeance partisane : les mondes franco-vénézuéliens de la réélection de Hugo Chávez (2012) »,

Critique internationale, 2013/2 №59, p. 119-132. DOI : 10.3917/crii.059.0119

Distribution électronique Cairn.info pour Presses de Sciences Po.

(C) Presses de Sciences Po. Tous droits réservés pour tous pays.

La reproduction ou représentation de cet article, notamment par photocopie, n'est autorisée que dans les limites des conditions générales d'utilisation du site ou, le cas échéant, des conditions générales de la licence souscrite par votre établissement. Toute autre reproduction ou représentation, en tout ou partie, sous quelque forme et de quelque manière que ce soit, est interdite sauf accord préalable et écrit de l'éditeur, en dehors des cas prévus par la législation en vigueur en France. II est précisé que son stockage dans une base de données est également interdit. 


\section{focus}

\section{Du nomadisme idéologique à l'allégeance partisane : les mondes}

\section{franco-vénézuéliens de la réélection de Hugo Chávez (2012)}

par Fabrice Andréani

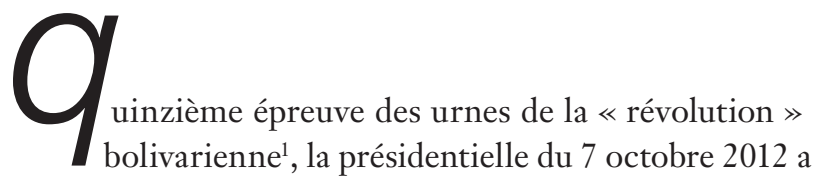
alimenté un casus belli médiatique global. Si l'état de santé du vainqueur, aujourd'hui décédé, a pu l'exacerber, la cristallisation pré-électorale des flux d'informations sur ce pays d'à peine 30 millions d'habitants ne date pas d'hier. À la fois catalyseur et relais d'une polarisation singulière, la médiatisation des

1. Ayant remporté 20 régions sur 23 en décembre 2012, puis gagné la présidentielle de 2013, le chavisme compte 16 victoires sur 17 scrutins depuis 1998. Nous écrivons sans guillemets bolivarien (étiquette non univoque) et avec guillemets « révolutionnaire »; en italique processus, synonyme de « révolution » bottom up et par les urnes (la « voie chilienne» de Salvador Allende (1970-1973) distincte de la « voie cubaine » armée) et chavisme, ensemble mouvant, situé, d'allégeances au chef. 
scrutins des années 2000 a dessiné une nouvelle « exception vénézuélienne »² sur la scène internationale. Cette image s'est nourrie, outre des joutes oratoires dans lesquelles se distinguait son représentant en chef, des controverses entre ses partisans et ses opposants qu'une quantité inhabituelle de médias (pan) américains et européens, notamment français, se sont empressés de couvrir. Ce faisant, divers exégètes - journalistes, intellectuels et experts - de ce régime politique non identifié ${ }^{3}$ ont souvent endossé les rôles de pro- ou d'anti-chavistes. Certains n'y consentaient pas nécessairement, d'autres les ont incarnés plus aisément que les rares élus qui s'y risquaient. Toujours est-il que ces mises en scène ont reproduit une doxa implicite qui a structuré leur coprésence sur les plateaux (radio)télévisés et dans les tribunes des grands quotidiens. Selon cette doxa, le rite électoral, ignoré à l'étranger du temps du «Venezuela Saudita » ${ }^{4}$ des années 1960-1980 - cet îlot démocratique et prospère dans un océan d'autoritarisme -, recèlerait aujourd'hui des enjeux autrement considérables. De fait, la généralisation de l'attention à son égard confère a priori à l'isoloir vénézuélien le crédit dû à une authentique épreuve de réajustement de l'« opinion publique », à une réduction inédite du « décalage entre le monde social tel qu'il est et ce qu'il devrait être pour satisfaire aux attentes morales des personnes $»^{5}$. Cependant, après quinze ans de vie politique sans alternance, chacun doute des dispositions de l'adversaire à respecter la vox populi une fois élu. Par ailleurs, la richesse du sous-sol national, première réserve mondiale de pétrole faisant du pays l'un des principaux fournisseurs des États-Unis, octroierait aux électeurs et aux élus une influence transfrontalière démesurée, parce qu'affranchie des lois de la realpolitik. En témoignent les spéculations sur l'éventualité d'une issue violente à l'annonce des résultats du scrutin ou du décès du « candidat de la vie » : c'était le destin de la région, du continent, voire de l'humanité qui se jouait là.

Nous prenons le parti de renverser ce métadiscours, géopolitique et prospectif, pour en analyser les conditions sociales de possibilité et les implications. Plutôt que d'imputer des enjeux extranationaux à une situation dont l'extrême sensibilité serait acquise ${ }^{6}$, nous explorerons les modalités de globalisation de cette campagne et leurs incidences sur la configuration des luttes politiques

2. Expression empruntée à Edgardo Lander, qui évoquait ainsi la stabilité de la situation politique vénézuélienne avant le milieu des années 1980. Edgardo Lander, «Venezuelan Social Conflict in a Global Context », Transnational Institute, mars 2005.

3. « Fascisme du $\mathrm{XXI}^{\mathrm{e}}$ siècle », « autoritarisme électoral », « populisme autoritaire », « démocratie représentative », «participative » et/ou « sociale » : j'ai recensé toutes ces définitions en parcourant les sessions rattachées à la Venezuelan Studies Section de la Latin American Studies Association (LASA) lors de son congrès international à San Francisco du 22 au 26 mai 2012.

4. Courant jusqu'au contre-choc pétrolier de 1983, ce terme fait référence aux richesses pétrolières de l'Arabie Saoudite.

5. Luc Boltanski, De la critique. Précis de sociologie de l'émancipation, Paris, Gallimard, 2009, p. 56.

6. Sur ce biais analytique, voir Nicolas Guilhot (ed.), The Invention of International Relations Theory: Realism, the Rockefeller Foundation, and the 1954 Conference on Theory, Columbia, Columbia University Press, 2011. 
vénézuéliennes à la veille et au lendemain de l'élection. Étant donné la bipolarité de cet espace-temps au Venezuela, relier la survisibilité de cadrages globalisants à leurs usages partisans locaux implique de soumettre les pratiques discursives et sous-jacentes correspondantes à un examen à la fois critique et compréhensif. Compte tenu de la multitude de sites matériels ou virtuels de (dé)légitimation publique du chavisme de par le monde et de leurs échelles d'imbrication, une réduction de focale s'impose. Nous nous concentrerons donc sur les circulations d'idées entre les champs médiatiques, politiques et (para-)académiques français et vénézuéliens. En analysant les parcours et les quotidiens militants et professionnels des entrepreneurs de causes francovénézuéliennes, dans le double contexte du boom pétrolier et de l'austérité européenne, nous serons mieux en mesure d'interpréter le sens et la portée au local de leurs interactions multiples, plus ou moins visibles et publicisées? Nous resituerons tout d'abord la transnationalisation des engagements polarisés autour des élections démocratiques bolivariennes dans la trajectoire historique du pétro-État. Ici, le vote est pensé à l'aune de son exact contraire : le coup d'État raté contre Chávez. L'événement met journalistes, intellectuels et experts au cœur d'une dispute opposant deux « systèmes de division du monde $\gg^{8}$; systèmes réifiés, via l'étranger, par les effets conjugués de l'hégémonie antirévolutionnaire et des victoires des gauches régionales. Au Venezuela, cette géopolitique imaginaire du vote, entretenue par des logiques multisituées et hétéronomes ${ }^{9}$, constitue un vecteur de disqualification des dissensions au sein et aux marges des deux coalitions, et ce, in fine, au profit des clans qui dominent le chavisme.

\section{Polarisation et globalisation d'un vote de crise}

Tributaires des aléas de l'économie-monde ${ }^{10}$, les porte-parole de la « révolution » bolivarienne et de l'Alliance bolivarienne pour les peuples de notre Amérique (ALBA-TCP) ${ }^{11}$ n'érigent pas moins ces dernières en « alternatives au

\footnotetext{
7. Les productions parues durant la campagne (officielle de juin à octobre 2012) sont tirées de la mailing list hebdomadaire « Le Venezuela dans la presse française » du service Communication de l'ambassade du Venezuela à Paris. Nous en avons testé l'exhaustivité sur Google une trentaine de fois en trois ans. Le tri opéré repose sur des observations, entretiens et analyses de discours réalisés entre le Venezuela et l'Europe de 2003 à 2012.

8. Jean Leca, « Le repérage du politique », Projet, 71, janvier 1973, p. 11-24.

9. Pierre Bourdieu, «Les conditions sociales de la circulation internationale des idées », Actes de la recherche en sciences sociales, 145 (5), 2002, p. 3-8.

10. Edgardo Lander, Margarita Lopez-Maya, «El socialismo rentista de Venezuela ante la caída de los precios petroleros internacionales », dans Birgit Daiber (ed.), La Izquierda en el Gobierno. Comparando América Latina y Europa, Bruxelles, Rosa Luxembourg Foundation, 2009. En 2012, le pétrole a généré 95 \% des exportations et $33 \%$ du PIB.

11. Les autres membres de l'Alliance-Traité de commerce des peuples sont : Cuba, la Bolivie, l'Équateur, le Nicaragua, Antigua-et-Barbuda, la Dominique, Saint-Vincent-et-les-Grenadines et le Honduras (jusqu'au coup d'État de 2009 contre Manuel Zelaya). Ses dirigeants la présentent comme un contre-projet à la Zone de libreéchange des Amériques.
} 
néolibéralisme $»^{12}$, voire au « système métabolique du Capital $»^{13}$. Candidat d'une « troisième voie $»^{14}$ matinée d'une refonte constitutionnelle en 1998, Hugo Chávez a fini par revendiquer la rupture du monopole « euro-américain $\gg$ de l'innovation démocratique ${ }^{15}$. Or, s'il a flirté avec la guérilla et tenté un putsch avant d'opter pour la voie des urnes, c'est en triomphant d'un putsch adverse que le président élu est consacré Comandante.

\section{De la guerre civile larvée à la lutte de classement}

« Président le plus élu au monde », Chávez est également le «miraculé » d'une éviction manu militari aussi brève que spectaculaire, avalisée par George W. Bush et José Maria Aznar. Arrêté le 11 avril 2002, après la télédiffusion par l'opposition d'un montage dans lequel on voyait «ses » francstireurs abattre des manifestants, il est libéré deux jours plus tard, grâce à la mobilisation de ses partisans descendus par millions des barrios pour réclamer son rétablissement à la tête de l'État, la suppression du « gouvernement de transition » et la levée du silence imposé aux médias ${ }^{16}$. L'entreprise de ces quelques militaires, patrons, élus, syndicalistes et journalistes matérialise une temporalité révolutionnaire qui n'existait jusque-là que dans l'imaginaire de certains partisans de la Constitution bolivarienne votée par référendum en $1999^{17}$ : le processus est considéré comme étant bel et bien « en marche» puisqu'il est mis en péril par l'« oligarchie ». La crise politique qui précède l'élection de Chávez refait ainsi surface alors même que les engagements du candidat de 1998 étaient réalisables au regard de la hausse du cours du brut ${ }^{18}$. Mais Chávez sait tirer parti du coup d'État, comme des manœuvres successives de l'opposition. Une grève pétrolière de plusieurs mois (2002-2003), déclenchée par la hiérarchie de l'entreprise nationale Petróleos de Venezuela (PDVSA) et cause d'une récession ponctuelle, entraîne le licenciement d'environ 20000 cadres « saboteurs », annonçant ainsi la reprise en main votée par l'Assemblée en 2001. En 2004, le référendum de révocation du président

12. Cette formulation renvoie à la célèbre expression de Margaret Thatcher : « There is no alternative » (TINA).

13. Hugo Chávez, « Discours à la Conférence des Parties de l’ONU sur le climat », Copenhague, 16 décembre 2009.

14. Malgré son parcours gauchisant, le candidat se référait explicitement à la « Troisième Voie » de Tony Blair et Anthony Giddens.

15. Immanuel Wallerstein, L'universalisme européen. De la colonisation au droit d'ingérence, Paris, Demopolis, 2008.

16. Des dessins animés passent en boucle après la lecture en direct de la fausse lettre de démission de Chávez, et l'annonce de la suspension des mandats électifs et de l'investiture de Pedro Carmona, chef du syndicat patronal Fedecámaras. Des tractations entre les deux camps sont encadrées jusqu'en 2005 par le Centre Carter. Jennifer McCoy, Francisco Diez, International Mediation in Venezuela, Washington D.C., United States Institute of Peace, 2011.

17. Cette Constitution institue la République bolivarienne du Venezuela et la « démocratie participative et protagonique $\gg$.

18. De 7 dollars en 1998, il atteint 120 dollars en 2008. Fernando Coronil, « Chávez's Venezuela: A New Magical State? », dans June C. Erlick (ed.), «The Chávez Effect », ReVista - Harvard Review of Latin America, 8 (1), 2008, p. 3-4. 
se transforme en plébiscite ${ }^{19}$, tandis qu'en 2005 le boycott des législatives par l'opposition, à la suite de discriminations à l'embauche de citoyens proréférendum à des postes de fonctionnaires, a pour conséquence l'élection d'une Assemblée « rouge » à $80 \%$. Si elle participe à nouveau aux scrutins suivants, l'opposition multiplie ses griefs à l'égard du pouvoir : le chavisme est accusé de contourner le « non » au référendum constitutionnel de $2008^{20}$ et de bénéficier d'un redécoupage territorial avant les législatives de $2010^{21}$. Partant, les usages concurrents de la crise aux présidentielles de 2006 et de 2012 déclinent des téléologies symétriques ${ }^{22}$ : la violence passée et/ou future des chavistes expliquerait celle des opposants, et vice versa. Le coup d'État raté de 2002 ferait écho à celui, également raté, du Movimiento Bolivariano Revolucionario 200 du 4 février 1992, dont Chávez avait endossé la « responsabilité » à l'écran tout en « prédisant » son retour ${ }^{23}$. Ce coup d'État, devenu entre-temps le Jour de la Dignité nationale, devait venger les martyrs de la plus sanglante des émeutes que l'Amérique ait connues depuis les années 1970 : le Caracazo du 27 février 1989, « événement fondateur » du processus ${ }^{24}$. En 2012, le candidat du Gran Polo Patriótico (GPP), emprisonné de 1992 à 1994 et en 2002, continuait de se poser en victime de l'« oligarchie impérialiste » et de son « héritier », le candidat de la Mesa de la Unidad Democrática (MUD), Henrique Capriles Radonski ${ }^{25}$. L'ordre d'avant 1999 était toujours ramené à ses deux péchés, l'un fondateur et l'autre mortel : le Pacte de Punto Fijo (1958), qui a exclu l'extrême gauche du jeu électoral ${ }^{26}$ pendant dix ans, et le massacre du Caracazo (1989). De son côté, Capriles jouait de son (bref) emprisonnement pour avoir co-occupé l'ambassade de Cuba en avril $2002^{27}$. Visé par un attentat la veille du début de la campagne

19. Décidée sur pétition de $20 \%$ des inscrits du corps électoral, la procédure de révocation des élus à mimandat date de cette Constitution.

20. Le « paquet socialiste » est saucissonné : un autre référendum est organisé et gagné en 2009 sur la seule question de la reconductibilité des mandats (dont le présidentiel), et les propositions clés sont votées par l'Assemblée hégémonique.

21. Suivi du vote d'une (troisième) Ley habilitante de 18 mois avant l'installation de la nouvelle Chambre, à $48 \%$ MUD : le président peut prendre des mesures $\mathrm{d}^{\prime} \ll$ urgence » (humanitaires) ; mais les lois clés requièrent les deux tiers à l'Assemblée nationale.

22. Mêlant étiologie, naturalisme et héroïsme. Michel Dobry, Sociologie des crises politiques, Paris, Presses de Sciences Po, 1983, p. 47.

23. Il négocie 30 secondes d'antenne pour « éviter un bain de sang », précisant que le MBR-200 n'a échoué que « pour l'instant ». Un autre putsch échoue en novembre.

24. Émeutes suscitées par l'imposition par le FMI d'un « plan d'ajustement structurel ». Paula VasquezLezama, « Le Caracazo (1989) vingt ans après : de l'économie morale à l'instrumentalisation politique de l'émeute au Venezuela », Revue internationale de politique comparée, 17, février 2010, p. 127-142. Le président visé était Carlos Andrés Pérez, héraut du retour de la (Gran) Venezuela Saudita de son premier mandat (1974-1979).

25. Au double sens du terme, politique et patrimonial, puisque son père est un des hommes d'affaires les plus riches du pays.

26. Et non pendant quarante ans (selon le pouvoir). Le pacte permet l'alternance entre Acción Democrática (sociaux-démocrates avec Romuldo Bettencourt et Pérez) et COPEI (démocrates-chrétiens). Rafael Caldera (COPEI) propose l'amnistie dès 1968.

27. Capriles, ex-maire de la circonscription où se trouve l'ambassade, dit l'avoir protégée, comme les élus chavistes qu'il a fait arrêter. Dernier d'une littérature déjà abondante, le livre Abril sin censura de l'ex-ambassadeur cubain German Otero Sanchez est lancé par le journal El Correo del Orinoco pour les dix ans du coup d'État. 
mais refusant la protection des services secrets, il a ravivé les rumeurs relatives à l'omniprésence d'agents cubains dans le pays ${ }^{28}$, tandis que les chavistes rappelaient les exactions de la police politique de la Quatrième République (1958-1999) ${ }^{29}$. Les médias sont les relais les plus fidèles de cette opposition caricaturale entre les « résistants à l'Empire » emmenés par Cuba et les garants de la « bonne gouvernance » représentés par les États-Unis et l'Union européenne. Ils en sont également la principale pierre d'achoppement, via l'épineuse question de la « liberté d'expression ». Compte tenu de leur rôle dans les coups d'État ratés de 1992 et de 2002, les deux camps les conçoivent comme des nœuds de liaison entre les réseaux militants populaires et la « communauté internationale ». Pour l'élection de 2012, un contentieux autour des temps de parole audiovisuels a très vite entaché la campagne. Capriles a menacé de ne pas signer la charte du Conseil national électoral (CNE) : selon lui, la suspension de l'émission dominicale Aló Presidente, dans laquelle Chávez « éduquait » ses concitoyens (et ministres), ne compensait pas le maintien, durant cette période, d'une autre curiosité locale, héritée des présidents « capitalistes », les cadenas présidentielles, allocutions d'une durée indéterminée et dont la retransmission est obligatoire, que la MUD dénonçait comme des clips de campagne déguisés. Au même moment, la chaîne de télévision privée Globovisión recevait une amende, en vertu de la loi sur la « responsabilité médiatique », pour avoir couvert des émeutes dans une prison de façon « partiale ». Cette sanction a rappelé l'affaire du non-renouvellement en 2007 de la licence hertzienne de la chaîne RCTV (Radio Caracas Televisión Internacional), porte-parole de la tentative de putsch de 2002 et par la suite d'appels à la destitution extra-électorale de « Chávez l'orang-outang ». Câblée à partir de 2007, RCTV a été transformée en « télé participative » d'État sous le nom de $T V E S^{30}$.

\section{Journalistes, intellectuels et experts dans une campagne importée}

La recherche de cautions démocratiques dans les pays du Nord est typique des situations de crises politiques au Sud. Or la quantité de titulaires de cartes de presse, de diplômes universitaires et/ou de galons de militants, disposés à

\footnotetext{
28. Voir par exemple Elizabeth Burgos[-Debray], « Paralelismos cubanos en la revolución bolivariana », Revista Venezolana de Ciencia Politíca, 29, enero-junio (janvier-juin) 2006, p. 39-71.

29. La DISIP (aujourd'hui SIBIN) serait responsable d'une centaine d'exécutions de transfuges de la guérilla dispersés dans divers barrios urbains (1968-1989). Entretien avec une avocate pénale, Mérida, juillet 2007.

30. «Te ves ( (tu te vois), acronyme de Televisora Venezolana Social. Adeline Joffres, « Démocratie participative et médias : jeux d'influence au Venezuela », L'ordinaire latino-américain, 210, septembre-décembre 2008, p. 193-215. Les chavistes estiment que la loi copie la régulation européenne. Venevisión, la chaîne hertzienne du milliardaire Gustavo Cisneros, bénéficie d'un pacte de non-ingérence avec l'État.
} 
investir dans la cause qui nous intéresse ici a été impressionnante ${ }^{31}$. Depuis 2002, la France et l'Espagne ont fourni un grand nombre de ces passeurs d'idées, alimentant ainsi des luttes pré-existantes au sein de leurs gauches respectives. En France, la critique « anti-autoritaire » (voire « antitotalitaire ») à l'encontre de Chávez, qui émane d'anciens « soixante-huitards » plutôt favorisés, domine largement la presse de centre gauche. En 2012, l'« effet Mediapart » a pu inciter Libération à diversifier ses sujets ou ses sources, mais le pluralisme se trouve plutôt du côté du Figaro ou de La Croix. Au Monde, l'anti-chavisme quasi inconditionnel va de pair avec la rareté des enquêtes in situ. On « correspond » souvent de Bogotá ou de Rio de Janeiro, ou bien l'on importe, via El País, des Unes d'opposition de Caracas (El Nacional, El Universal, TalCual ${ }^{32}$, dans lesquelles les partenariats du Venezuela avec des rogue States nourrissent le fantasme d'un nouvel « Axe du Mal $\gg$ Caracas-La Havane-Téhéran-Damas-Minsk (éventuellement Moscou) ${ }^{33}$. Paulo Paranagua, nommé « responsable Amérique latine » au Monde une fois revenu de ses désillusions guerrilleras en Argentine, a traité Chávez d'« antisémite » : le qualificatif d'« apatride » dont celui-ci a gratifié Capriles (au sens d'« impérialiste $»)$ signifierait $«$ juif errant $»^{34}$. Cette $\ll$ ligne $»$, qui se rapproche de celle d'une MUD encore hostile au «Vietnam Nord $»^{35}$ et de son porte-voix parisien associant Chávez à Bachar-al-Assad ${ }^{36}$, est aussi radiotélévisée ${ }^{37}$. En témoigne, entre autres, une émission de service public où Franz-Olivier Giesbert a lancé trois fois à un diplomate vénézuélien, à propos de l'imagerie révolutionnaire, «ça fait un peu Corée du Nord, non ? $»^{38}$.

Assimilant cette presse à l'ultraconservateur Miami Herald ${ }^{39}$, le chavisme « global » a mobilisé ses nombreux réseaux. Depuis l'appel de Chávez au « socialisme du XXI ${ }^{\mathrm{e}}$ siècle » au Forum social mondial de Porto Alegre en 2005, puis à une

31. Sur les effets culturels (et scientifiques) des circulations « humanistes » des social scientists, voir Jonathan Friedman, « Des racines et (dé)routes. Tropes pour trekkers », L'Homme, 156, octobre-décembre 2000, p. 187-206. 32. En dix ans, environ 10 articles du Monde sur 1000 correspondent à une enquête in situ. Marie Delcas (Bogotá) nous a dit en avril 2012 : «Jean-Pierre Langellier vivant avec une Vénézuélienne, il couvre désormais le Venezuela [depuis Rio] ».

33. Pour une critique de ce cadrage, voir Bertrand Badie, La diplomatie de connivence. Les dérives oligarchiques du système international, Paris, La Découverte, 2011.

34. Les grands-parents juifs de Capriles ont fui la guerre et se sont réfugiés au Venezuela. « Dérapages antisémites de la campagne de Chávez au Venezuela », Le Monde, 6 septembre 2012. Sur Paulo Paranagua et la Fracción Roja de la guérilla argentine du PRT-ERP, voir Daniel Bensaïd, Une lente impatience, Paris, Stock, 2004. 35. Relevé dans une expertise du parti de Capriles, Primero Justicia, cf. infra.

36. Voir Francisco Merino, « M. Mélenchon fait de la désinformation sur le Venezuela », Mediapart, 31 août 2012. 37. En raison de l'influence du quotidien sur les producteurs de contenus radiotélévisés, comme d'ailleurs sur ceux du Point, des Échos et du Nouvel Observateur.

38. « Parlez-moi d'ailleurs : le phénomène Chávez », La Chaîne parlementaire 10 juin 2012, avec Maurice Lemoine (Le Monde diplomatique), Farid Fernández (ambassade vénézuélienne), Renée Fregosi (Paris 3, Institut des hautes études de l'Amérique latine - IHEAL-CREDA, membre du PS) et François-Xavier Freland (Radio France Internationale, auteur du pamphlet Qui veut la peau d'Hugo Chávez? Paris, Cherche Midi, 2012).

39. « De la mafia anticastriste » (selon Chávez), dont le «terroriste » (avéré) Luís Posada Carriles, réfugié aux États-Unis. 
« Cinquième Internationale » à Belém en 2009, une partie de la gauche européenne qui a un pied dans l'« altermondialisme » et l'autre dans le jeu électoral a vu dans Chávez et l'ALBA-TCP l'occasion de rejouer une carte institutionnelle mise en sourdine à la création du $\mathrm{FSM}^{40}$. On trouve dans ce courant des témoins clés du coup d'État de 2002, filmé en live par les réalisateurs irlandais de The Revolution will not be televised, documentaire indémodable d'initiation « alter » à la cause bolivarienne ${ }^{41}$. Maurice Lemoine, chargé du Venezuela au Monde diplomatique jusqu'en 2012, «n'[a] pas attendu Chávez pour [s']y intéresser » : il vadrouille dans la région depuis trois décennies ${ }^{42}$. Avec Ignacio Ramonet, biographe de Fidel Castro, il fait partie des intervenants favoris des débats et événements en tous genres organisés par l'ambassade vénézuélienne ou le Cercle bolivarien ${ }^{43}$. Rempart contre une désinformation antibolivarienne systématisée, le Monde diplomatique est devenu le canal de convergence de la vulgarisation de la critique « citoyenne » des médias, portée par Acrimed $^{44}$, et de la promotion d'une « Sixième République » par une gauche « re-fondationnelle », les yeux rivés sur le « laboratoire latino ». Christophe Ventura, de l'Association pour la taxation des transactions financières (ATTAC) et de la Commission internationale du Parti de Gauche (PG), conclut de ses voyages au Venezuela et dans la région qu'« ici aussi [la crise de la dette aidant] le centre gauche et la droite seront balayés par une troisième force, tôt ou tard » avec la prescience d'une même « bataille médiatique » à la clét5

D'où une hybridation notable entre les médias « alternatifs » étrangers, locaux (Radio del Sur, Aporrea), binationaux (Venezuelainfos ${ }^{46}$ ) et d'État (ALBA-TV, Telesur). Se revendiquant d'Antonio Gramsci, les experts bolivariens voient dans cette production intellectuelle la marque d'une « contre-hégémonie », foisonnante et « hétérogène », plutôt que d'une idéologie nationale-étatique ${ }^{47}$. « Pacifique mais armée », récusant le « socialisme réel », la « révolution » est « anti-doctrinaire », « non alignée », à l'instar du Cuba des années 1960-198048.

40. Sur le courant « post-alter », voir Fabrice Andréani, « Indiens dans la ville, bourgeois dans la jungle l'ALBA enlisée sur le front climatique », Mouvements, 70, été 2012, p. 59-69.

41. Observations à Paris, Lyon, Caracas, Mérida, Barcelone, Madrid, Rome, 2003-2010. Documentaire de Kim Bartley et Donnacha O'Briain, Power Pictures, 2002.

42. Entretien avec Maurice Lemoine, Paris, janvier 2010. M. Lemoine, Chávez Presidente !, Paris, Flammarion, 2005. 43. Observation d'une vingtaine de conférences, tables rondes et ateliers à Paris (Maison de l'Amérique latine, Sénat, Ubifrance, Fête de l'Humanité, 2009-2012). Eurosur, ONG de Bernard Cassen, en assure souvent la logistique. I. Ramonet a dirigé le Monde diplomatique jusqu'en 2008 et créé Mémoire des luttes. Cassen et lui ont fondé ATTAC et le FSM.

44. Acronyme du site web « Action critique des médias ». Serge Halimi, Les nouveaux chiens de garde, Paris, Raisons d'agir, 1997, et le film éponyme de Gilles Balbastre et Yannick Kergoat (Épicentre Films, 2011).

45. Entretien avec Christophe Ventura, Paris, décembre 2011. Actif à Mémoires des luttes, Eurosur, aux stands Venezuela / ALBA des « Fêtes de l'Huma », C. Ventura enseigne la géopolitique à Paris 8.

46. De Thierry Deronne (PC belge, Vive TV), lié à Bella Ciao et au Grand Soir (http://www.larevolucionvive.org). 47. Entretiens à l'Universidad Bolivariana (Mérida, Caracas) et à l'Instituto de Investigación Cientifíca (Caracas, Paris), 2008-2011.

48. Steve Ellner, «Venezuela's Bottom-Up Participation: Advances and Downsides », Congrès de la LASA, cité. 
Cette image est encensée, en Europe ou aux États-Unis, par des universitaires titulaires disposés à s'exprimer en public aux côtés de cadres (et de vacataires) ouvertement chavistes, en raison certes d'affinités idéologiques,_mais aussi (et parfois uniquement) pour contrer la survisibilité de pairs anti-chavistes ${ }^{49}$. En France, le Nouveau Parti anticapitaliste $(\mathrm{NPA})^{50}$, qui a réaffirmé son soutien à Chávez aux côtés du Front de Gauche, n'ignore pas les « dérives » bureaucratiques de ce « réformisme » recensées par la revue de la Quatrième internationale, Inprecor $^{51}$. Au Centre tricontinental de Louvain-la-Neuve (tiers-mondiste), à Recherches internationales (liée au Parti communiste) et même au Monde diplomatique, plusieurs articles vont dans ce sens ${ }^{52}$.

Toutefois, les postures les plus visibles - et les plus commentées sur la Toile - sont sans conteste les moins nuancées, comme cet extrait des « Carnets de voyage » de Jean Ortiz dans L'Humanité : «Au Venezuela, beaucoup de gens connaissent et respectent Jean-Luc Mélenchon et Ignacio Ramonet $»^{53}$. À l'approche du jour J, tandis que le Front de Gauche latino prépare la « fête électorale » du 7 octobre, Alexandre Adler et Daniel Cohn-Bendit dissertent abondamment sur les « régimes nauséabonds » de Messieurs Chávez, Orban et Loukatchenko ${ }^{54}$. Enfin, les aficionados s'affrontent à coups de tribunes dans Le Monde : l'une est signée R. Fregosi, membre du Parti socialiste mais ici strictement « directrice de recherche au CNRS »; l'autre, J.-L. Mélenchon et I. Ramonet ${ }^{55}$. Celle du journaliste Marc Saint-Upéry, pétrie de critiques de la «boli-bourgeoisie » (bolivarienne), n'est commentée à la « gauche de la gauche » que via des mailing lists d'initiés ${ }^{56}$.

49. Invitée à nombre de débats bolivariens, Jeanette Habel de l'IHEAL-CREDA paraît plus nuancée qu'une R. Fregosi radicalement pro-MUD. À comparer aussi Olivier Compagnon, Julien Rebotier, Sandrine Revet (dir.), Le Venezuela au-delà du mythe. Chávez, la démocratie, le changement social, Paris, Les Éditions de l'Atelier/ Éditions ouvrières, 2009, ouvrage dépolarisé, et Stephen Launay, Chávez-Uribe, deux voies pour l'Amérique latine?, Paris, Buchet Chastel, 2010, thèse $\mathrm{du}$ « fascisme bonapartiste ».

50. François Sabado (ancien de la Ligue communiste révolutionnaire) a relayé l'appel à la « Cinquième internationale ». Voir aussi Daniel Bensaïd, Olivier Besancenot, Prenons parti. Pour un socialisme du XXI siècle, Paris, Mille et une Nuits, 2009.

51. Franck Gaudichaud, « Les tensions du processus bolivarien : nationalisme populaire, conquêtes sociales et capitalisme rentier », Inprecor, 588-589, 2012, et Contre-Temps, 10 décembre 2012.

52. Bernard Duterme (dir.), État des résistances dans le Sud. Amérique latine, Paris, Syllepse, 2012 ; Thomas Posado (dir.), « Les gauches latino-américaines au pouvoir », dossier thématique, Recherches internationales, 93, 2012 ; S. Ellner, «Au Venezuela, un chavisme sans Chávez ? », Le Monde diplomatique, septembre 2012.

53. J. Ortiz (PCF) a co-organisé à l'Université de Pau un colloque quasi intégralement pro-Chávez, en présence d'officiels du régime. Observations, 25-26 mars 2010. L'ensemble des « Carnets » (titre du film sur le Che) est accessible sur http://www.humanite.fr/monde/le-blues-le-vague-lame-la-saudade-la-anoranza-desrevolutionnaires-502387.

54. À la conférence « Les enjeux des élections présidentielles au Venezuela » de la revue Building, Maison de l'Amérique latine, $1^{\text {er }}$ octobre 2012. Outre R. Fregosi, était annoncée une « diplomate vénézuélienne » dont le titre, usurpé, a été démenti par l'ambassade.

55. R. Fregosi, «H. Chávez, autocrate ou démocrate ? », Le Monde, 4 octobre 2012 ; J.-L. Mélenchon, I. Ramonet, «H. Chávez, un homme diffamé », Le Monde, 5 octobre 2012.

56. Observations, ATTAC et PG, Marseille, Paris, octobre 2012. Marc Saint-Upéry, « Un anti-modèle à gauche », Le Monde, 4 octobre 2012 ; « Mélenchon-Chávez, une idylle mal venue », Fournal du Mauss, 4 octobre 2012. 


\section{Le pluralisme à l'épreuve de l'État « multipolaire »}

Au Venezuela, c'est le soutien du Parti de Gauche qui est le mieux accueilli par le chavisme enclin à la même illusion médiatique ${ }^{57}$. Facilité par l'ancrage en Europe des exilés qui ont, pour la plupart, fui les dictatures du Cône $\mathrm{Sud}^{58}$, le cosmopolitisme de gauche se nourrit autant sinon plus d'asymétries socioéconomiques, professionnelles et générationnelles que d'« homologies de position $»^{59}$. Les parcours de certains jeunes Vénézuéliens d'origine modeste, formés à Cuba comme aux États-Unis ou en Europe, contrastent en effet avec les auto-expatriations parfois erratiques d'(ex-)étudiants européens en Amérique latine. Moins visibles que leurs aînés formés au nomadisme militant dans les années 1960, et peu rétribués, ces derniers ont des savoir-faire qui peuvent tout de même se révéler utiles à l'occasion.

\section{Des cosmopolitismes statocentrés}

Soutenu dans ses entreprises électorales par le président équatorien Rafael Correa ainsi que par une centaine de cadres gouvernementaux et de mouvements associés latinos ${ }^{60}, \mathrm{~J}$-L. Mélenchon s'est rendu au Venezuela après sa double défaite, présidentielle et législative. Dans son blog, il relate ce 14 juillet 2012 où il a contemplé avec Chávez et Ramonet une « immense marée rouge » depuis un char de campagne. Ressentant « la même émotion » que pendant ses meetings, il se félicite que l'orchestre ait joué La Marseillaise : Chávez « a compris la portée universelle de la Révolution française $»^{61}$. L'éloge apparaît déjà dans Le Grand Bon en arrière (El gran salto pa'tras), tourné par cinq Français vivant au Venezuela qui reviennent dans leur pays filmer «sous Sarkozy [les dégâts de] quarante ans de néolibéralisme », et dans lequel I. Ramonet déplore le vote « contre-nature » de ses concitoyens pauvres «manipulés par les médias $»^{62}$. À la suite du chef du PG, Alexis Corbière, François Delapierre et Corinne Morel-Darleux exposent à la télévision vénézuélienne leur vision de la crise européenne et de la « Triade néolibérale $»^{63}$, et ce malgré l'avis contraire de militants qui s'« autocensurent » le temps

\footnotetext{
57. Pour une critique de l'élitisme de gauche, voir Jacques Rancière, Le spectateur émancipé, Paris, La Fabrique, 2008. 58. Comme le Chilien Andrés Bansart, docteur en civilisation hispanique de l'Université de Tours et directeur de l'Universidad de Latinoamérica y del Caribe, et également de tous les débats.

59. Yves Dezalay, « Les courtiers de l'international. Héritiers cosmopolites, mercenaires de l'impérialisme et missionnaires de l'universel », Actes de la recherche en sciences sociales, 151-152, 2004, p. 4-35.

60. La lettre « Ici comme là-bas vive la révolution citoyenne » est publiée sur le site Place au Peuple 2012 le 15 juin, celle du « Collectif latino-américain de soutien » sur le site web du Grand Soir le 19 mars.

61. Voir http://www.jean-luc-melenchon.fr/2012/07/16/no-volveran/ (consulté le $1^{\text {er }}$ septembre 2012).

62. El gran salto pa'tras, TVES / Altermedia, 2011. Leur dernier film traite des campagnes de 2012 dans les deux pays.

63. «Dirigente socialista francés : elección del 7-0 interesa a izquierda global », Ciudad Caracas, 29 septembre 2012.
} 
d'un vote les engageant malgré eux $^{64}$. Entre-temps, d'autres membres du PG corédigent entre Caracas et Paris un Kit élections vénézuéliennes, conçu pour leurs concitoyens sujets au «bombardement médiatique ». Sous-titré « Pourquoi la victoire de Chávez est aussi [la] nôtre », ce kit d'« éducation populaire » résume ainsi « la longue et triste nuit néolibérale »: «Quarante ans (...) d'une oligarchie (...) liée aux multinationales (...) et sourde vis-à-vis des besoins de la population $\gg^{65}$.

Co-auteur du Kit, Romain Mingus s'est illustré par une autre publication sortie au plus fort de la campagne à destination, cette fois, des Vénézuéliens indécis ou abstentionnistes ${ }^{66}$. Téléchargeable depuis les plateformes (para)gouvernementales, El programa de la MUD dissèque en 166 pages les 1337 articles de l'accord de la coalition d'opposition ${ }^{67}$. Dans la foulée, le Parti socialiste unifié du Venezuela (PSUV) révèle une expertise confidentielle interne à Primero Justicia, le parti conservateur de Capriles. La série de « mesures d'urgence » envisagées face à la « probable contraction de la demande de pétrole », et dont l'authenticité n'a pas été démentie, confirme point par point les analyses de R. Mingus : décentralisations en trompe-l'œil (privatisations), dénationalisations, restitution de terres expropriées, restauration de l'autonomie de la Banque centrale et des taux de changes flexibles. On y sonne également le glas de ce que d'aucuns appellent les « cadeaux aux pauvres »: le programme Petrocaribe, qui consiste à vendre du pétrole à terme et à taux bas à la quasi-totalité des États caribéens, qui en échangent une partie contre des aliments ou des médicaments. Plus grave, si elle prétend « pérenniser » les misiones phares du proceso $^{68}$, improvisées avec Cuba dans le contexte préréférendaire de 2004, la MUD préconise la suspension pure et simple de Mercal (alimentation) et de la (récente) Gran Misión vivienda (logement, hyperactive en 2011-2012). S'agissant des hydrocarbures au Venezuela, les experts de Primero Justicia sont aussi élusifs que ceux du cabinet présidentiel : un autre texte serait « trop sensible » pour circuler en campagne. Diverses voix internes à la MUD réclament un débat « public et responsable » sur le document révélé : quatre micro-partis se retirent de la MUD et le député William Ojeda est expulsé de Un Nuevo Tiempo (centre-gauche de la coalition) ${ }^{69}$.

R. Mingus, militant du PG, contribue ainsi au délitement de la coalition à un

\footnotetext{
64. Observations, Paris, Marseille et Lille, septembre-octobre 2012.

65. Eduardo Meneses et al., Kit militant élections vénézuéliennes, Paris, PG-FDG, 2012.

66. Il fait partie des premiers « révolutionnaires français» auto-expatriés entre 2002 et 2005, comme les auteurs du documentaire El gran salto pa'tras.

67. «El programa MUD no favorece a los venezolanos », El Correo del Orinoco, 21 août 2012 ; Romain Mingus, El programa de la MUD : Análisis de los Lineamientos de Gobierno de la MUD, Caracas, Barrio Alerta, 2012.

68. Carlos Romero, « La política exterior de Venezuela. La respuesta de la oposición democrática », Nuevos Mundos Mundos Nuevos, 30 janvier 2012.

69. Sous prétexte d'une affaire de détournement de fonds. « Continua desintegración comando campaña derecha », Patria Grande / Aporrea, 19 septembre 2012.
} 
mois du scrutin. Son initiative aurait pu le mener ailleurs, mais elle lui donne dans la nouvelle Mecque de l'« internationalisme » un statut qu'il n'aurait pu imaginer en France. Un temps traducteur «invisible » des discours de Chávez, il est finalement « cité » par le Comandante à la télévision, dans un discours retransmis depuis un stade plein à craquer ${ }^{70}$. Reconnu « chercheur et sociologue » au Venezuela, mais considéré en France comme un « journaliste français vivant au Venezuela », il incarne cette possibilité de mobilité ascendante - toujours symbolique, parfois matérielle - escomptée par de nombreux étudiants européens devenus experts intermittents, et dont certains bénéficient au niveau local de la légitimité que leur confère la fréquentation d'établissements comme Sciences Po, La Sorbonne ou l'EHESS ${ }^{71}$.

\section{L'économie morale de l'u anti-impérialisme »}

En fin de campagne, les habitudes refont surface : face à une MUD acculée à fantasmer sur une fraude dont la possibilité est écartée par des experts internationaux ${ }^{72}$, le GPP brandit le spectre d'un « sabotage putschiste » en cas de victoire, ce qui achève de neutraliser les critiques de début de campagne au sein du PSUV. Exprimées en marge du Forum de São Paulo (juillet 2012), celles-ci dénoncent les conditions de la coopération aux frontières qui entourent le récent « réchauffement » diplomatique entre la Colombie et le Venezuela. Les déportations extrajudiciaires des (ex-)combattants des FARC et de l'ELN depuis le Venezuela sont en effet devenues légion, hypothéquant la «paix » colombienne négociée depuis le début de l'année 2012 à La Havane ${ }^{73}$. Et s'il a enterré le projet d'installation de nouvelles bases américaines, le Président colombien Juan Manuel Santos n'a pas résolu l'affaire des « faux positifs », ces centaines de faux « guérilleros » colombiens exécutés par des (para)militaires avides de primes des deux pays ${ }^{74}$. Enfin, depuis quelque temps, les dizaines d'assassinats impunis d'indigènes par des latifundistes, en territoire frontalier à haute valeur minière (charbon), ont rejoint la liste des tabous d'un chavisme très souvent $\ll$ en campagne $\gg^{75}$.

D'aucuns expliquent ce réchauffement par les projets binationaux d'oléoducs pour le transit vers la Chine, à laquelle le Venezuela rembourse ses dettes (croissantes) en pétrole. À cet égard, de plus en plus de réfractaires et de

\footnotetext{
70. Selon les termes d'une témoin locale, entretien via Skype, octobre 2012.

71. Observations et entretiens, Caracas, Mérida, Paris, Madrid, 2006-2012.

72. Jimmy Carter : « Des 92 élections que nous avons expertisées, je dirais que le processus électoral vénézuélien est le meilleur au monde ». Marc Weisbrot, «Why the US Demonises Venezuela's Democracy », The Guardian, 3 octobre 2012.

73. «Joaquín Pérez Beccera : una condena efectiva pero "cibernética” », ANNCOL, 23 septembre 2012.

74. « Piedad Córdoba insiste en que MinDefensa aclare los llamados "falsos positivos" », Caracol, 9 septembre 2012.

75. « Continua la lucha Yukpa por sus territorios », El Libertario, 68, diciembre-enero (décembre-janvier) 2013.
} 
dissidents estiment que le gouvernement a plus ménagé l'« ouverture pétrolière » imposée par le FMI qu'il ne l'a révolutionnée. La consécration constitutionnelle du caractère «national » du « pétrole de tous les Vénézuéliens » s'accompagne en effet de celle des « entreprises mixtes » : PDVSA s'associe aux « oligarchies » jusque dans l'exploitation du brut, et non plus seulement dans sa commercialisation. Selon l'ambassadeur français Jean-Marc Laforêt, « pour Total tout cela [les nationalisations et hausses d'impôts] n'est qu'une petite goutte dans un lac de stabilité ( ), ils sont là pour cent ans $\gg^{76}$. Or, s'il n'y a jamais eu autant de firmes étrangères sur le sol national, comme dans la faille pétrolifère de l'Orénoque, ces firmes sont en concurrence pour « semer le pétrole » (sembrar el petróleo), selon une devise centenaire, c'està-dire financer : des raffineries Petro-ALBA de Cuba aux projets ruraux de « développement endogène intégral », des satellites chinois aux radios « communautaires », et autres « coopératives », en passant par des nationalisations « stratégiques » (mines, télécommunications, électricité). L’ultradépendance alimentaire et industrielle demeure pourtant inchangée et les dévaluations de mise après les élections, comme au début de l'année 2013. Il n'empêche que les électeurs-actionnaires de la rente «magique » jugent le président sortant à l'aune de dividendes que l'opposition semble hypothéquer, plutôt qu'à celle de la corruption notoire des clans qui l'entourent ${ }^{77}$.

Aux côtés d'un primo-partenaire étasunien dépourvu d'ambassadeur mais déclaré « neutre $»^{78}$ et d'un Brésil ouvrant à la « révolution » la porte du MERCOSUR et fermant à la MUD celle de la Cour interaméricaine des droits de l'homme ${ }^{79}$, la France reflète bien l'ambivalence de cet « anti-impérialisme ». Après que Jean-Marc Ayrault a disqualifié J.-L. Mélenchon sur la base de son chavisme à la « rentrée politique », Benoît Hamon inaugurait à Caracas la première visite du gouvernement en Amérique latine. À cette occasion, les chavistes consacrés par le vote populaire se sont enthousiasmés de l'ouverture annoncée d'une usine Renault ${ }^{80}$, annonce en revanche peu commentée par leurs relais français, ennemis déclarés des « patrons voyous ». La multiplication de ces unités de production hybrides exacerbe les conflits syndicaux dans les

76. Entretien avec Jean-Marc Laforêt, Caracas, juillet 2010.

77. F. Coronil, The Magical State. Nature, Money, and Modernity in Venezuela, Chicago, University of Chicago Press, 1997. Dans le doute, Chávez a remplacé in extremis Diosdado Cabello, ex-vice-président (président intérimaire et candidat à son décès), par Nicolás Maduro, moins communément traité de « voleur » dans les milieux populaires.

78. Le Président Obama à Romney l'accusant de ménager un « dictateur » : « Au vu de ce qu'il a fait ces dernières années, M. Chávez ne constitue pas une menace pour les États-Unis », Reuters, 24 septembre 2012.

79. L'entrée du Venezuela au Mercosur suit la mise hors jeu par le Brésil, l'Argentine et l'Uruguay du Paraguay où un coup d'État a renversé Fernando Lugo. Voir « Une figure de l'altermondialisme défend la CIDH », Le Monde, 4 septembre 2012.

80. Entretien par Skype avec une invitée à l'ambassade, 25 novembre 2012. « Hamon à Caracas », Les Échos, 24 novembre 2012. 
entreprises nationalisées, et/ou sous « contrôle [bureaucratique]-ouvrier $»^{81}$, tandis que la globalisation de la discipline électorale les relativise, au profit d'un chavisme « dominé » à l'étranger. Face à un Mélenchon rallié par la Gauche alternative (ex-NPA) et qui vitupère contre la « diabolisation » de ses « amis », la minorité soutenant au NPA la candidature du syndicaliste dissident Orlando Chirino est invisible. Le sont également, face à un Noam Chomsky ou une Naomi Klein, les libertaires relayant les données de l'ONG PROVEA qui attestent un regain contestataire au sein du chavisme depuis 2006 (occupations, grèves, manifestations) et sa répression (para)policière, même si celle-ci est bien moindre qu'avant $1999^{82}$.

\begin{abstract}
À l'heure où les médias établissent des analogies entre les funérailles de Chávez et celles de Staline ${ }^{83}$, et à l'ombre d'une bipolarité consolidée, la globalisation des luttes politiques vénézuéliennes ré-enchante les « cultures matérielles » de ses protagonistes et de ses bénéficiaires ${ }^{84}$ : celles qui distinguent, d'un côté, des opposants bien établis, de l'autre, de jeunes Européens en quête d'un El Dorado révolutionnaire ; mais aussi celles qui rapprochent les classes dominantes du pétro-État et des pays « émergents ». C'est toute l'ambivalence d'une révolution institutionnalisée, à l'image de son héritier Nicolás Maduro, ouvrier autodidacte devenu ministre des Affaires étrangères, à la fois bureaucrate syndical redouté et diplomate respectée ${ }^{85}$.
\end{abstract}

Fabrice Andréani est attaché temporaire d'enseignement et de recherche en science politique au CERAPS/Université Lille 2, et doctorant au Centre d'études et de recherches internationales (CERI-Sciences-Po/CNRS, Paris). andreani.fabrice@gmail.com

\footnotetext{
81. Voir T. Posado, « Ni victoire ni défaite : le chavisme en crise », Recherches internationales, 89, juillet-mars 2011, p. 23-40.

82. Voir Octavio Alberola, passé à la Confédération nationale du travail de Perpignan au détour d'une tournée espagnole : « La verdad sobre Venezuela », El Libertario, 68, diciembre-enero (décembre-janvier) 2013. Sur Chomsky, voir Rafael Uzcátegui, Venezuela : révolution ou spectacle?, Paris, Spartacus, 2010.

83. Observation in situ, «JT francophone », France 24, 16h30-17h00, Issy-les-Moulineaux, 8 mars 2012.

84. Jean-François Bayart, Jean-Pierre Warnier (dir.), Matière à politique. Le pouvoir, les corps et les choses, Paris, Karthala, 2004.

85. Le dauphin désigné de Chávez est élu le 14 avril 2013 avec 50,5\% des voix. Alors que la MUD avait accepté la victoire de Chávez du 7 octobre 2012 - avec 54 \% des voix -, la justesse de celle de Maduro lui permet de contester officiellement les résultats, avec l'appui des États-Unis. Ne se contentant pas du recompte légal de $54 \%$ des bureaux choisis aléatoirement, et des dizaines d'expertises internationales, elle a obtenu du CNE un recompte « total ».
} 